\title{
A detailed study of the course, branching pattern and dimensions of the uterine and vaginal arteries and their branches
}

\begin{abstract}
Introduction: Despite the critical role of the uterine artery (UA) and vaginal artery (VA) in the blood supply to the pelvic organs and cavity, there is a paucity of descriptions of their origin, course, and dimensions.
\end{abstract}

Aim: The aim of the study was to present a detailed account based on a cadaveric study on pelvic halves of 31 female embalmed cadavers belonging an Indian population.

Methods: A total of 31 female cadavers (62 pelvic halves) in the age group of 34-75 years belonging an Indian population were dissected.

Results: There were no variations in the origin, course, distribution and length of the UA and VA and their branches. However, in a significant number of cadavers, the diameter of the UA was smaller than normal, while the corresponding VA in these same cadavers was larger than normal. Additionally, in other cadavers where the diameter of the UA was larger than normal, the diameters of the VA was smaller than normal.

Conclusion: Thus, uterus received complementary blood supply from the UA and VA. However, a more definitive pattern of blood supply to the uterus could not be established due to a limited number of cadavers. Further, since adequate studies from different population groups were not available, a more detailed description of the branching patterns and dimensions of the UA and VA and their branches in different population groups could not be formulated.

Keywords: pelvic halves, physiological processes, vaginal artery, maternal pathologies, childbirth
Volume 6 Issue 6 - 2019

\author{
A Vasantha Lakshmi,' Mustafa Vali SK, \\ Venugopala Rao B, ${ }^{2}$ Nirmala Palayanthan, ${ }^{2}$ \\ Joydeep D Chaudhuri ${ }^{3}$ \\ 'Department of Anatomy, Nimra Institute of Medical Sciences, \\ India \\ 2MAHSA University, Malaysia \\ ${ }^{3}$ School of Occupational Therapy, College of Health Sciences, \\ Husson University, USA
}

Correspondence: Joydeep Dutta Chaudhuri, College of Health and Pharmacy, Husson University, College Circle, Bangor, Maine, 0440I,Tel 207852 8747, Fax 207973 I06I, Email chaudhuri@husson.edu

Received: November 13, 2019 | Published: December II, 2019
Abbreviations: UA, uterine artery; VA, vaginal artery; EIA, external iliac artery; IIA, internal iliac artery; CIA, common iliac artery; SD, standard deviation

\section{Introduction}

The blood supply of a region is determined by functional considerations, such that structures participating in a common activity tend to receive their blood supply from the same arterial source., ${ }^{1,2}$ Since, this inevitably make simultaneous demands on a limited blood supply, the pattern of arterial branching is fundamental to the understanding of the efficiency of blood supply to a region. ${ }^{3,4}$ In this context, the concentration of anatomical structures within the closely packed confines of the pelvis makes the study of the vascular patterns of the uterine artery (UA) and vaginal artery (VA) particularly critical in the elucidation of the physiological processes and the management of pathological conditions of pelvic. ${ }^{1,5}$

Pregnancy is a physiological state which imposes a considerable demand on uterine blood. ${ }^{6}$ Since increased uteroplacental blood flow is necessary for a normal outcome of pregnancy, dysfunction of uterine blood flow has been implicated in the causation of fetal and maternal pathologies. ${ }^{2,7}$ An adequate blood supply is normally accomplished during pregnancy, by an increase in the diameter of the uterine artery (UA), without a corresponding thickening of the arterial wall. From a clinical standpoint, in rare occasions bilateral
UA ligation has been used in the management of intractable uterine bleeding during childbirth. ${ }^{3,5,8}$

In addition, pathological conditions of the female reproductive tract in general and the uterus in particular, are among the most common reasons for gynaecological referrals. ${ }^{4,7}$ Of these, uterine fibroids are one of the most common uterine tumors, and symptomatic fibroids is one of the most common indications for hysterectomy. ${ }^{8}$ The blood supply through the UA facilitates the growth of these fibroids, and hence a reduction of blood supply is utilized to reduce their size. ${ }^{9}$ Therefore, arterial embolization of the UA is commonly employed as a preoperative adjunct or as an alternative to surgery in the treatment of uterine fibroids. ${ }^{1,4,10}$ In addition, hysterectomies are often carried in post-menopausal women to manage large uterine fibroids or malignancies. ${ }^{11}$ Thus, the regulation of blood flow through the UA is the basis for many of the therapeutic interventions that are commonly employed for management of conditions of the uterus. ${ }^{12}$

Hence, considering the clinical implications an accurate description of the vascular pattern of the UA and VA and their branches will lead to better preoperative investigation and diagnosis, and increased success of minimally invasive surgery. However, determination of the accurate blood flow volume measurements in the UA are subject to many limitations that include, but are not limited to, anatomic issues on the sampling site, ${ }^{13}$ and variations of collateral blood flow from uterine, vaginal and ovarian arteries. ${ }^{3,8,14}$ There is a paucity of detailed 
reports of the hemodynamics of the uterus and vagina despite their critical role in the blood supply to the pelvic cavity. ${ }^{9}$

\section{Blood supply of the pelvic cavity}

The internal iliac artery (IIA) is one of the terminal branches of the common iliac artery (CIA), the other terminal branch being the external iliac artery (EIA). While the EIA supplies the lower extremity, the IIA supplies the pelvic viscera, perineum, pelvic wall and gluteal region through its various branches..$^{15}$ The IIA terminates into anterior and posterior divisions, typically anterior to the sacroiliac joint. The anterior divides into visceral branches that supply the pelvic organs and the parietal branches that supply the muscles of the gluteal region and upper thigh. ${ }^{2,7,9}$ All the branches from the posterior division are parietal, and supply the ventral and dorsal aspects of the posterior abdominal wall and the gluteal region. ${ }^{16}$

The UA is one of the visceral branches of the anterior division of the IIA. Each UA passes anterior to the pelvic part of the ureter, and courses from the lateral to the medial direction along the inferior part of the broad ligament of the uterus. It then descends to the level of the isthmus of the uterus, where it bifurcates into ascending and descending branches. The ascending branches course along the margin of the uterus, and anastomose with branches of the ovarian artery, which is a direct branch of the abdominal aorta. These branches supply the upper part of the uterus, part of uterine tube, ovary, other contents of the broad ligament of the uterus, and the urinary bladder. ${ }^{5,17}$ The descending branches of the UA anastomose with ascending branches from the VA and supply the uterine cervix and vagina. In addition, the UA also gives rise to intramural branches that arise from the medial aspect and are called the arcuate arteries. The terminal branches of the arcuate arteries frequently anastomose with those of the contralateral side to provide blood supply to the myometrium of the uterus.

The VA is also a branch of the anterior division of the IIA, and is often considered to be a homolog of the inferior vesical artery in males. ${ }^{14,16,18}$ The VA arises slightly superior and posterior to the origin of each UA and descends inferiorly to supply fundus of the urinary bladder, adjacent part of the rectum and upper part of the vagina. The VA also has ascending branches which anastomose with the descending branches from the UA, and supply the cervix and upper part of the vagina.

\section{Aim of study}

A patchwork of information exists regarding details of the vascular anatomy of the UA and VA and its branches. Hence, this study aims to provide a detailed account of the level and mode of origin and termination, length and mean external diameter of these arteries and their branches in an Indian population. It also aims to compare the results of this study with previously reported anatomical, physiological surgical, and radiological studies on these arteries.

\section{Material and methods}

The study was performed, following institutional approval, on randomly chosen embalmed cadavers prepared for student dissection in the Department of Anatomy, NRI Medical College, and Guntur, India. A total of 31 female cadavers (62 pelvic halves) in the age group of 34-75 years belonging an Indian population were studied. None of the cadavers displayed any signs of obvious pelvic disease, injury, vascular intervention or surgery. The present study was confined to the IIA and its branches that are located only within the pelvis.
All the cadavers were embalmed with a modified Larssen solution in preparation for teaching and research purposes within the Department of Anatomy, NRI Medical College, India. The modified Larssen solution included $100 \mathrm{~mL}$ of $10 \%$ formalin, $400 \mathrm{~mL}$ glycerine, $200 \mathrm{~g}$ chloral hydrate, $200 \mathrm{~g}$ sodium sulphate, $200 \mathrm{~g}$ sodium bicarbonate, $180 \mathrm{~g}$ sodium chloride in the final working solution of $9.5 \mathrm{~L}$ of distilled water. ${ }^{5}$ The solution was maintained at $\mathrm{pH} 9$ by using Sodium borate as a buffer. ${ }^{19}$ An average of 6.5-7.1L was infused arterially in each cadaver, using a pulsed infusion at a rate of $60-70$ pulses per minute, and the whole injection was performed over $30-45 \mathrm{~min}^{3}{ }^{3}$

In each embalmed cadaver a vertical incision was made in the anterior abdominal wall from the xiphoid process of the sternum to the symphysis pubis. Transverse incisions were then made from the umbilicus to the right and left sides of the abdominal wall. The two upper skin flaps of abdominal wall were reflected upwards and laterally and the lower two skin flaps were reflected downwards and laterally. The pelvic viscera were retracted laterally to expose the UA and VA and its branches. The other individual branches of the IIA were identified and then removed, along with the internal iliac vein and its tributaries were identified and then removed to obtain a clear exposure of the UA and VA.

The lengths of UA and VA were measured using a Fowler 54101-175 Poly-Cal Digital Calliper (resolution: $0.01 \mathrm{~mm}$ accuracy: $0.02 \mathrm{~mm}$ ). The external diameters of these arteries were measured using a Mitutoyo 753-1966 Digimatic Digital (resolution: 0.005mm accuracy: $0.01 \mathrm{~mm}$ ). All measurements were taken without stretching or compressing the artery, and external diameters of the branches were recorded at their point of origin from the IIA, as described by Babuccu et al. ${ }^{4}$ At least two measurements of each vessel were performed, and the mean value was accepted as the mean external diameter of the vessel. To minimize inter-observer error, $25 \%$ of the measurements were also re-taken by a separate, independent individual.

All statistical analyses were carried out using SPSS software, version 11.0 (SPSS Inc.; Chicago, Ill). The values are presented as mean $\pm \mathrm{SD}$; and range. The Chi-squared test was used to analyse differences between the right and left sides, while continuous data were compared using the Student t-test. A $p$-value of $\leq 0.05$ was considered significant.

\section{Observations}

A total of 31 female cadavers (62 pelvic halves) belonging to an Indian population were studied. All dimensions of the UA and VA are presented as the mean \pm standard deviation (SD) and range. In conformity with previous literature, an artery with a dimension of 1 $\mathrm{SD}$ or more below the mean value, was operationally defined to be smaller than normal. Similarly, a vessel with a dimension of $1 \mathrm{SD}$ or more above the mean value, was considered to be larger than normal. ${ }^{7,20}$

\section{Internal iliac artery}

In all specimens, on both sides, the IIA originated as a terminal branch of the CIA and was located anterior to sacroiliac joint, and descended posteromedially into the lesser pelvis. It had a normal origin, course, and distribution, and then terminated into anterior and posterior divisions. The anterior division continued towards the ischial spine, while the posterior division exited the pelvic cavity through the suprapiriform foramen. The mean length of the IIA on the left side was $3.68 \pm 0.16 \mathrm{~cm}$ (range: $3.63-4.06 \mathrm{~cm}$ ), and $3.54 \pm 0.22 \mathrm{~cm}$ (range: 3.27 - 
$3.79 \mathrm{~cm})$ on the right side. The mean external diameters of IIA were $10.43 \pm 0.35 \mathrm{~mm}$ (range: $10.17-10.98 \mathrm{~mm}$ ) and $10.35 \pm 0.29 \mathrm{~mm}$ (range: $10.19-10.58 \mathrm{~mm}$ ) on the left and right sides respectively. There were no significant differences in the mean lengths or external diameters of the IIA in between both sides of individual cadavers ( $p>0.05$ ), or among individual cadavers $(\mathrm{p}>0.05)$ (Table 1$)$.

\section{Uterine artery}

The UA originated from the anterior division of the IIA in all the cadavers, and had a tortuous course (Figure 1). It crossed the ureter at right angles and descended to the level of the internal os of the cervix, where it divided into ascending and descending branches. The ascending branches coursed superiorly along the margin of the uterus and continued medially along the base of the broad ligament, and supplied the upper part of the uterus, part of uterine tube, urinary bladder and terminal part of ureter. Branches of the ascending division of the UA anastomosed with branches from the ovarian artery within the broad ligament and supplied the contents of the broad ligament of the uterus. In addition, descending branches of the UA were observed along both sides of the supra vaginal part of cervix that anastomosed with the ascending branches of the VA. These branches supplied the uterine cervix and vagina. The arcuate arteries could also be observed arising from the medial aspect of the UA on both sides and supplied muscular branches to the anterior and posterior aspects of the uterus. No variations were observed in the origin, course and distribution of the UA.

The mean length of the UA was $4.32 \pm 0.11 \mathrm{~cm}$ (range: $4.19-4.45$ $\mathrm{cm}$ ) on the left side, and $4.29 \pm 0.09 \mathrm{~cm}$ (range: $4.18-4.43 \mathrm{~cm}$ ) on the right side. There were no significant differences in the lengths of the UA between the right and left sides, or among individual cadavers $(\mathrm{p}>0.05)$. The mean external diameter of UA was $1.38 \pm 0.42 \mathrm{~mm}$ (range: $0.91-1.99 \mathrm{~mm}$ ) on the left side, and $1.39 \pm 0.38 \mathrm{~mm}$ (range: 0.99 $1.88 \mathrm{~mm})$ on the right side. In $12.90 \%(\mathrm{n}=8)$ cadavers the diameters of the UA were smaller than normal (1 SD or less below the mean external diameter), while in $9.68 \%(n=6)$ cadavers the diameters of the UA were larger than normal ( $1 \mathrm{SD}$ or less above the mean external diameter). No significant differences in any dimensions of the UA were observed between the left and right sides of individual cadavers $(\mathrm{p}>0.05)$ (Table 2).

Table I Dimensions of the internal iliac artery

\begin{tabular}{llll}
\hline & \multicolumn{1}{l}{ Left side (Mean \pm SD) } & Right side (Mean \pm SD) & \multirow{2}{*}{ P-value } \\
\cline { 2 - 3 } & $\mathbf{( R a n g e )}$ & $\mathbf{( R a n g e )}$ & \multirow{2}{*}{0.063} \\
\hline Length of internal iliac artery $(\mathrm{cm})$ & $3.68 \pm 0.16$ & $3.54 \pm 0.22$ & \multirow{2}{*}{$0.07 \mid$} \\
External diameter of internal iliac artery $(\mathrm{mm})$ & $(3.63-4.06)$ & $(3.27-3.79)$ & $10.35 \pm 0.29$ \\
\hline
\end{tabular}

Table 2 Dimensions of the uterine artery

\begin{tabular}{llll}
\hline & Left side (Mean \pm SD) & Right side (Mean \pm SD) & P-value \\
\cline { 2 - 3 } & $($ Range) & (Range) & 0.071 \\
\hline Length of uterine artery $(\mathrm{cm})$ & $4.32 \pm 0.11$ & $4.29 \pm 0.09$ & 0.066 \\
External diameter of uterine artery $(\mathrm{mm})$ & $(4.19-4.45 \mathrm{~cm})$ & $(4.18-4.43 \mathrm{~cm})$ & $1.39 \pm 0.38$ \\
\hline
\end{tabular}

Table 3 Dimensions of the vaginal artery

\begin{tabular}{llll}
\hline & \multicolumn{1}{c}{ Left side (Mean \pm SD) } & Right side (Mean \pm SD) & P-value \\
\cline { 2 - 3 } & $\mathbf{( R a n g e )}$ & $\mathbf{( R a n g e )}$ & 0.069 \\
\hline Length of vaginal artery $(\mathrm{cm})$ & $6.57 \pm 0.15$ & $6.76 \pm 0.18$ & \\
External diameter of vaginal artery $(\mathrm{mm})$ & $(6.39-6.87 \mathrm{~cm})$ & $(6.44-6.76 \mathrm{~cm})$ & 0.067 \\
& $1.33 \pm 0.54$ & $1.48 \pm 0.57$ & $(0.82-2.28 \mathrm{~mm})$ \\
\hline
\end{tabular}




\section{Vaginal artery}

The observations of the VA are also based on the same 62 female pelvic halves that have been previously described. In all the cadavers it originated from the anterior division of the IIA, and descended towards the vagina (Figure 1). The VA divided into ascending and descending branches that supplied both surfaces of the vagina, and adjacent parts of the urinary bladder and rectum. The ascending branches of the VA could be seen anastomosing with descending branches of the UA and supplied the lower part of the cervix and upper part of the vagina. The descending branches of the VA supplied the lower part of the vagina and adjoining tissues. No anatomical variations were recognized in the origin, course and distribution of the VA.

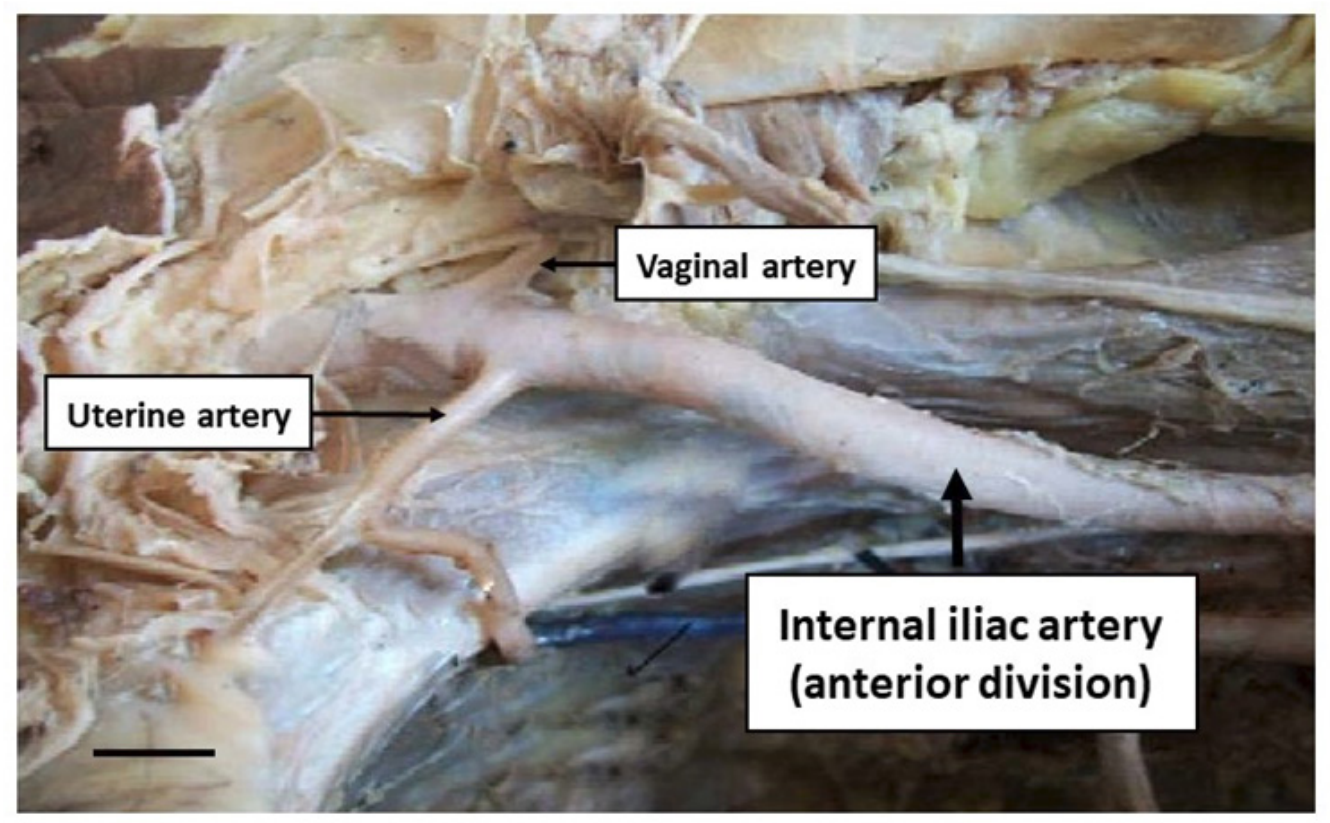

Figure I The uterine artery and vaginal artery arising from the anterior division of the internal iliac artery. Scale bar represents Icm.

The mean length of the VA was $6.57 \pm 0.15 \mathrm{~cm}$ (range: 6.39 $6.87 \mathrm{~cm}$ ) on the left side and $6.76 \pm 0.18 \mathrm{~cm}$ (range: $6.44-6.76 \mathrm{~cm}$ ) on the right side. There were no significant differences in the lengths of the VA between the right and left sides ( $\mathrm{p}>0.05)$. The mean external diameter of the VA was $1.33 \pm 0.54 \mathrm{~mm}$ (range: $0.76-2.13 \mathrm{~mm}$ ) on the left side and $1.48 \pm 0.57 \mathrm{~mm}$ (range: $0.82-2.28 \mathrm{~mm}$ ) on the right side. However, in $6.45 \%(\mathrm{n}=4)$ cadavers the diameters of the VA were smaller than normal (1 SD or less below the mean external diameter), while in $8.06 \%(\mathrm{n}=5)$ cadavers the diameters of the UA were larger than normal (1 SD or less above the mean external diameter). No significant differences in any dimensions of the VA were observed between the left and right sides of individual cadavers ( $p>0.05)$ (Table $3)$.

\section{Comparisons of the diameters of vaginal and uterine arteries in individual cadavers}

Since the external diameters of the UA and VA displayed significant variations, further analysis of each individual pelvic halve was carried out. In cadavers where the diameter of the UA was smaller than the mean external diameter $(\mathrm{n}=8)$, the diameter of the VA was also larger than the mean external diameter in $6.52 \%(n=5)$ cadavers. Correspondingly, in the cadavers where the diameter of the UA was larger than the mean external diameter $(n=6)$, the diameter of the VA was smaller than the mean external diameter in $6.45 \%(n=4)$ cadavers. However, no significant differences $(p>0.05)$ in dimensions were observed between the left and right sides of any of these individual cadavers that showed variations.
Hence, while the UA and VA did not display any significant variations in their lengths, there were significant variations in the external diameters of the UA and VA. In a total of $14.52 \%(n=9)$ cadavers the mean external diameters of the UA and VA were antipodally smaller or larger.

\section{Discussion}

Mapping arterial variations and quantifying their dimensions is significant in both research and clinical settings, since they often serve as surrogates for determinates for vascular health..$^{16,21}$ Heterogeneity in arterial dimensions represents a spectrum, the extremes of which represent pathological changes. ${ }^{22}$ The existing data in the literature on the UA and VA and their branches has focused on the origin, course, distribution and dimensions with very limited information on bilaterally and racial variations. ${ }^{5}$ This underscores the need for reference databases to determine the efficiency of arterial circulation at a given anatomical point. ${ }^{5,11,23}$ Thus, the specific aim of this study was to compile normative data to add to the body of information regarding the origin, distribution and dimensions of the UA and VA and their branches.

Cadaveric derived information of specific arteries is fundamental to the interpretation of results of computerized angiographic studies. Methodological constraints can limit magnetic resonance angiography due to the limited resolution of smaller blood vessels within the pelvis, and the lack of bony landmarks. ${ }^{22}$ Conventional angiography of UA and VA have been reported to occasionally create a false impression 
of arterial occlusion within the pelvis because of slowing of blood flow through aberrant branches of the UA and VA. ${ }^{24}$ Similarly metal implants have also been reported to cause artefact formation in computerized tomography generated angiographic images. ${ }^{6,11,25}$ In addition, pelvic ischemia has at times been reported to be wrongly diagnosed because of erroneous placement of the catheter tip into aberrant branches of UA and VA. ${ }^{26}$ Lastly, since conventional Doppler is often limited in identifying deeply located arterial perforators, color Doppler is often used. ${ }^{22}$ However, the accuracy of color Doppler is dependent on human variations such as the experience of radiologist. ${ }^{17}$ Hence, a detailed cadaver-based identification is crucial in the management of bleeding in the pelvic cavity, avoidance of iatrogenic injury to the branches of IIA, treatment of uterine fibroids and the use of perforator flaps in reconstruction surgery.

All vascular dimensions in this study were recorded using electronic digital callipers which have been documented to have a high resolution and accuracy. ${ }^{6}$ In addition, the reliability of the methods of measurements used in this study have also been previously confirmed by three-dimensional digital image correlation (3D-DIC) studies, ${ }^{27}$ high resolution $\mathrm{B}$-mode ultrasound ${ }^{11,14}$ and direct in vivo measurements. ${ }^{12}$ The accuracy of the measurements in this study were further reconfirmed by comparing dimensions obtained using slide calipers with measurements using digital stereo images of arteries using digital CCD Point Grey cameras with an image resolution of $1028 \times 768$ pixels (data not shown).

Since this study involved the analysis of dimensions of blood vessels of embalmed human cadavers, concerns can be raised about how reflective these values are of the actual living population. Such concerns are most relevant when cadavers have been rapidly frozen resulting in shrinkage, ${ }^{9,19,23}$ or rapidly filled with latex resulting in expansion of blood vessels. ${ }^{6}$ In this study, an appropriate volume of a modified Larssen solution ${ }^{2,16}$ was used for embalming which has been shown to prevent fragmentation of both soluble and non-soluble proteins, ${ }^{9,11,20}$ and ensuring adequate fixation of lipids ${ }^{5}$ and nucleic acids in cadavers. ${ }^{5}$ This ensures that the linear non-load-deformation characteristic of blood vessels is maintained in cadavers..$^{24}$ In addition, the IIA and its branches contain a high percentage of elastic tissue ${ }^{13}$ and smooth muscle in the tunica media, ${ }^{11,24}$ and hence are not prone to collapse. ${ }^{5}$ Therefore, concerns regarding the effect of distortion and shrinkage in cadavers that have been embalmed with a modified Larssen solution are largely unfounded.

In this study no variations were observed in the origin or course of the UA or VA in any of the cadavers. This is in contrast to previous studies where the UA has been reported to originate from a common trunk along with the umbilical artery in $80.7 \%$ of cases, ${ }^{3}$ or arise directly from the IIA as a common trunk along with the umbilical artery in $13.16 \%$ of cadavers. ${ }^{17}$ There are also reports of the origin of the UA from the superior gluteal artery in $3.51-9.17 \%$ of cases. ${ }^{22,28}$ Further, no variations were observed in the course of the VA. The findings in this study are again in contrast to previous studies that reported that the VA has also been observed to arise as a common trunk with the inferior gluteal artery in $1.75 \%$ of cadavers, and directly from the OA in $0.88 \%$ of cases. ${ }^{9}$ However, it is possible that the lesser number of cadavers in this might have precluded the identification of variations of the UA or VA>.

These variations in the origin of the UA and VA, can be attributed to the uneven, ${ }^{5,11,22}$ and unpredictable ${ }^{11}$ growth of anastomotic blood vessels in the pelvic cavity during embryological development. ${ }^{9}$ The final arterial pattern of the pelvic cavity and lower extremity is derived from a selection of vascular channels that arise from the abdominal plexus and plexuses. ${ }^{20}$ During embryological development, the most appropriate channels connecting these two plexuses enlarge, while others retract and disappear. ${ }^{11,19}$ However, before the final appearance of the UA and VA as independent blood vessels the blood flow destined for the pelvis and lower extremities makes anomalous choices of source channels. ${ }^{14}$

The mean length of the UA was $4.32 \pm 0.11 \mathrm{~cm}$ (range: 4.19 $4.45 \mathrm{~cm}$ ) and $4.29 \pm 0.09 \mathrm{~cm}$ (range: $4.18-4.43 \mathrm{~cm}$ ) on the left and right sides respectively. The mean length of the VA on the left side was $6.57 \pm 0.15 \mathrm{~cm}$ (range: $6.39-6.87 \mathrm{~cm}$ ), and on the right side the mean length was $6.76 \pm 0.18 \mathrm{~cm}$ (range: $6.44-6.76 \mathrm{~cm}$ ). There were no significant differences in the lengths of the UA or VA between the right and left sides, or among individual cadavers $(p>0.05)$. The findings of this study are in agreement with previous studies where the length of the UA have been reported to be between $4.1-5.2 \mathrm{~cm}$, and $6.2-7.3 \mathrm{~cm}$ of the VA. ${ }^{3,11,24}$

The most striking observations in this study were the variations in the external diameters of the UA and the VA. In cadavers where the UA was bilaterally narrower than normal, the VA was bilaterally larger than normal. In addition, cadavers where the UA was wider than normal the VA was narrower than normal. This advances the suggestion that the UA and VA could be providing significant collateral blood supply to the uterus, then has been suggested in literature. This is clinically relevant as one of the approaches for the management of uterine conditions is by the restriction of the blood supply of the uterus. However, reports exist of the reoccurrence of uterine fibroids ${ }^{21,27}$ and even an increase in their sizes following occlusion of the UA. ${ }^{15,19}$ Based on the results of this study, the possibility exists that the diameter of the VA increased to provide more blood to the uterus following occlusion of the UA. This is plausible considering that a compensatory increase in blood flow in the ovarian artery has been reported in pathologic conditions of the uterus. However, though these vascular abnormalities were observed, due to the limited number of cadavers and a lack of complete medical history it could not be determined if the changes in the external diameters of the UA and VA were a compensatory mechanism or a physiological phenomenon.

In addition, the variations in the branching patterns observed in this study, and also reported in previous literature, also provide a possible reason for reports of the sudden onset of bleeding in the pelvic cavity without apparent cause following reconstructive surgeries in $2.2 \%$ to $34 \%{ }^{12}$ patients. Though dislodgement of occluding material at the bleeding site has been offered as an explanation, ${ }^{4}$ no definite evidence supporting this viewpoint has been reported. Hence it can be postulated that such unexpected postoperative bleeding may be due to the opening of aberrant branches of the UA and VA that could not be detected during initial angiographic procedures due to transient compensatory vasospasm, though no aberrant branches were observed in this particular study.

Hence, the variations observed in the UA and VA provides an insight into the blood supply of the pelvic cavity. It also underscores the need for a more detailed analysis of the branching patters of the UA and VA. 


\section{Limitations}

However, certain limitations exist regarding this study. Firstly, no detailed medical records were available about the cadaveric specimens. Hence, it could not be ascertained if any obstetrical or gynaecologic conditions could have resulted in the changes observed in the external diameter of the UA and VA. The use of a large sample of embalmed specimens with a detailed examination of medical records will yield more exhaustive information.

Again, while this study provides a detailed description of the origin, course, distribution and dimensions of the UA and VA and their branches, the extrapolation of this data to a larger and more universal population is restricted. This is primarily because this study was carried out on a relatively smaller number of cadavers. In addition, adequate comparisons within the Indian population, or any other demographic group was not possible due to the fragmented nature of the available information. Hence for a more detailed description of the UA and VA and their branches, studies on a larger number of cadavers from different population groups are necessary.

Finally, the variations that were observed in the external diameters of some of the branches of the UA and VA, could be attributed to physiological responses to the Body Mass Index (BMI) of an individual. However, the BMI could not be determined in these cadavers as they had already been perfused, and hence accurate measurements of the body weight were not possible. Hence, the values of the vascular dimensions could not be normalized to perform a correlation analysis, since calculations based on the BMI have been reported to produce the most accurate correlations. ${ }^{17}$

\section{Conclusion}

The findings in this study are clinically significant considering the vital role of UA and VA of providing collateral blood supply to the uterus. Since manipulation of the blood supply to the uterus is a common therapeutic intervention a description of the UA and VA and their branches, will facilitate the acquisition of better clinical results, and avoid iatrogenic complications due to untargeted embolization of the surrounding organs. Considering some of the limitations of computerized angiographic investigations, studies using embalmed human bodies are still vital in the elucidation of anatomicalmorphological features.

This is clinically significant in the management of conditions associated with these locations. The UA and VA have a critical role in providing blood supply to the pelvic organs. Hence In addition, as diagnostic and interventional angiographic procedures become more popular, such information would be valuable in the prevention of interruption of circulation.

\section{Author contributions}

A Vasantha Lakshmi study design; cadaveric dissection

SK Mustafa Vali: study design; cadaveric dissection; revision of manuscript

B Venugopala Rao: study design; writing and revision of manuscript

Nirmala Palayanthan: study design; writing and revision of manuscript
JD Chaudhuri: study design; statistical analysis; manuscript writing; revision of manuscript

\section{Acknowledgments}

The authors hereby acknowledge those who donated their bodies, and without whom this research would not have been possible.

\section{Conflicts of interest}

The authors declare no conflict of interest.

\section{References}

1. Ampatzidou F, Koutsogiannidis CP, Megalopoulos A, et al. Endovascular approach in chronic aortoiliac disease in patient undergoing coronary artery bypass surgery. Ann Card Anaesth. 2019;22(1):96-97.

2. Arfi A, Arfi-Rouche J, Barrau V, et al. Three-dimensional computed tomography angiography reconstruction of the origin of the uterine artery and its clinical significance. Surg Radiol Anat. 2018;40(1):85-90.

3. Athanaselis ED, Rountas C, Koutalos A, et al. MR angiogram confirms sustained blood flow in 1, 2 ICSR artery of vascularized bone grafting in scaphoid nonunion treatment. Eur J Orthop Surg Traumatol. 2018;29(2):343-348.

4. Babuccu O, Ozdemir H, Hosnuter M, et al. Cross-sectional internal diameters of radial, thoracodorsal, and dorsalis pedis arteries in children, relationship to subject sex, age, and body size. $J$ Reconstr Microsurg. 2006;22(1):49-52.

5. Donas KP, Inchingolo M, Cao P. Secondary procedures following iliac branch device treatment of aneurysms involving the iliac bifurcation, the pELVIS registry. $J$ Endovasc Ther. 2017;24(3):405-410.

6. Fang JF, Shih LY, Wong YC, et al. Angioembolization and laparotomy for patients with concomitant pelvic arterial hemorrhage and blunt abdominal trauma. Langenbecks Arch Surg. 2011;396(2):243-250.

7. Fujimoto A, Morimoto C, Hosokawa Y, et al. Suturing method as a factor for uterine vascularity after laparoscopic myomectomy. Eur $J$ Obstet Gynecol Reprod Biol. 2017;211:146-149.

8. Gorbach AM, Leeser DB, Wang H. Assessment of cadaveric organ viability during pulsatile perfusion using infrared imaging. Transplantation. 2009;87(8):1163-1166.

9. Gulik TM. New concepts in liver preservation, how the pendulum sways back. Liver Transpl. 2009;15(1):1-3.

10. Hassen-Khodja R, Batt M, Michetti C, et al. Radiologic anatomy of the anastomotic systems of the internal iliac artery. Surg Radiol Anat. 1987;9(2):135-140.

11. Hunter C, Moody L, Luan A, et al. Superior gluteal artery perforator flap, the beauty of the buttock. Ann Plast Surg. 2016;76 Suppl 3: S191S195.

12. Jeremias A, Kirtane AJ, Stone GW. A test in context, fractional flow reserve, accuracy, prognostic implications, and limitations. $J$ Am Coll Cardiol. 2017;69(22):2748-2758.

13. Jiang WX, Zhou ZE, Yan WG, et al. Unexpected hemorrhage of a rare vessel, a pubic branch of the external iliac artery, after laparoscopic radical prostatectomy, Case report. Medicine (Baltimore). 2017;96:e9357.

14. Kamenskiy A, Miserlis D, Adamson P. Patient demographics and cardiovascular risk factors differentially influence geometric remodeling of the aorta compared with the peripheral arteries. Surgery. 2015;158(6):1617-1627. 
15. Kawai K, Honma S, Koizumi M, et al. Inferior epigastric artery arising from the obturator artery as a terminal branch of the internal iliac artery and consideration of its rare occurrence. Ann Anat. 2008;190(6):541548.

16. Krishnamurthy A, Nayak SR, Khan S, et al. Anomalous channels draining the internal iliac veins, embryological and clinical significance. Rom J Morphol Embryol. 2007;48(1):71-73.

17. Leow $\mathrm{CH}$, Bazigou E, Eckersley RJ, et al. Flow velocity mapping using contrast enhanced high-frame-rate plane wave ultrasound and image tracking, methods and initial in vitro and in vivo evaluation. Ultrasound Med Biol. 2015;41(11):2913-2925.

18. Naguib NN, Nour-Eldin NE, Hammerstingl RM. Three-dimensional reconstructed contrast-enhanced MR angiography for internal iliac artery branch visualization before uterine artery embolization. J Vasc Interv Radiol. 2008;19(11):1569-1575.

19. Oshida S, Mori F, Sasaki M. Wall shear stress and T1 contrast ratio are associated with embolic signals during carotid exposure in endarterectomy. Stroke. 2018;49(9):2061-2066.

20. Palmer SK, Zamudio S, Coffin C, et al. Quantitative estimation of human uterine artery blood flow and pelvic blood flow redistribution in pregnancy. Obstet Gynecolec. 1992;80(6):1000-1006.

21. Stalder MW, Lam J, Allen RJ, et al. Using the retrograde interna mammary system for stacked perforator flap breast reconstruction, 71 breast reconstructions in 53 consecutive patients. Plast Reconstr Surg. 2016;137(2):265e-277e.
22. Sapmaz E, Celik H, Altungül A. Bilateral ascending uterine artery ligation vs. tourniquet use for hemostasis in cesarean myomectomy. A comparison. J Reprod Med. 2003;48(12):950-954.

23. Pelage JP, Soyer P, Le Dref O. Uterine arteries, bilateral catheterization with a single femoral approach and a single 5-F catheter--technical note. Radiology. 1999;210(2):573-575.

24. Schnapauff D, Russ M, Kröncke T, et al. Analysis of presurgical uterine artery embolization (PUAE) for very large uterus myomatosus; patient's desire to preserve the uterus; case series and literature review. Rofo. 2018;190(7):616-622.

25. Schoeman M, van Schoor A, Suleman F, et al. The validity of arterial measurements in a South African embalmed body population. Surg Radiol Anat. 2018;40(1):39-43.

26. Smith WJ, Upton E, Shuster EJ, et al. Patient satisfaction and disease specific quality of life after uterine artery embolization. Am J Obstet Gynecol. 2004;190(6):1697-1703.

27. Tajima S, Hoshina K, Oushik T, et al. Intimal sarcoma of the abdomina aorta and common iliac arteries presenting as epithelioid angiosarcoma of the skin, a case report. Int J Clin Exp Pathol. 2015;8(5):5856-5862.

28. Waliszewski MW, Redlich U, Breul V, et al. Surrogate and clinical endpoints for studies in peripheral artery occlusive disease, Are statistics the brakes? Pol Przegl Chir. 2017;89(2):39-48. 